\title{
Percepção de pacientes cirúrgicos sobre segurança e seu envolvimento no cuidado à saúde
}

\author{
Surgical patients' perception of safety and their involvement in health care \\ Percepción de los pacientes quirúrgicos sobre la seguridad y su participación en la atención médica
}

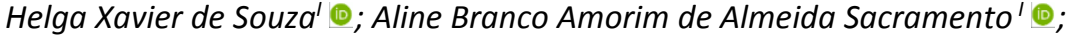

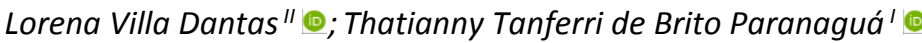

'Universidade de Brasília, Brasília, DF, Brasil; "Pesquisadora autônoma

\begin{abstract}
RESUMO
Objetivo: investigar a percepção do paciente cirúrgico sobre segurança do paciente e o seu envolvimento no cuidado à saúde durante a internação hospitalar. Método: estudo exploratório de abordagem qualitativa, com 14 pacientes em pós-operatório. Dados obtidos através de entrevistas semiestruturadas, realizadas em 2016 em um hospital de reabilitação, e analisados conforme o referencial de Bardin. Resultados: os participantes associaram segurança à estrutura física, confiança institucional, capacitação da equipe de saúde e humanização da assistência. O envolvimento se deu, principalmente, no processo de administração de medicamentos e em situações que divergiam do plano de cuidado conhecido pelo paciente. Conclusão: a percepção dos pacientes sobre segurança da assistência e erros em saúde foi associada a situações vivenciadas ou veiculadas na mídia. $O$ envolvimento dos pacientes ocorreu através de ações que se configuraram como barreiras para ocorrência de erros relacionados à assistência à saúde, legitimando seu papel como corresponsável pela segurança do cuidado.

Descritores: Segurança do Paciente; Participação do Paciente; Assistência Centrada no Paciente; Assistência à Saúde.
\end{abstract}

\section{ABSTRACT}

Objective: to investigate surgical patients' perceptions of patient safety, and their involvement in health care during hospitalization. Method: in this qualitative, exploratory study of 14 postoperative patients, data were obtained by semistructured interview in 2016 at a rehabilitation hospital, and analyzed taking Bardin as the theoretical framework. Results: the participants associated safety with physical structure, institutional trust, the health team's training, and humanized care. They were involved mainly in the medication administration process and in situations that diverged from the care plan known to the patient. Conclusion: patients' perceptions of health care safety and health care errors were associated with situations they experienced or which were broadcast in the media. Patient involvement occurred in actions constituted as barriers to the occurrence of health care errors, thus legitimizing their role as co-responsible for safe care.

Descriptors: Patient Safety; Patient Participation; Patient-Centered care; Delivery of Health Care.

\section{RESUMEN}

Objetivo: investigar las percepciones de los pacientes quirúrgicos sobre la seguridad del paciente y su participación en la atención médica durante la hospitalización. Método: en este estudio cualitativo, exploratorio de 14 pacientes postoperatorios, los datos se obtuvieron mediante entrevista semiestructurada en 2016 en un hospital de rehabilitación, y se analizaron tomando a Bardin como marco teórico. Resultados: los participantes asociaron la seguridad con la estructura física, la confianza institucional, la formación del equipo de salud y la atención humanizada. Se involucraron principalmente en el proceso de administración de medicamentos y en situaciones que divergían del plan de atención conocido por el paciente. Conclusión: las percepciones de los pacientes sobre la seguridad en la atención de la salud y los errores en la atención de la salud se asociaron con situaciones que vivieron o que fueron difundidas en los medios. La participación del paciente se produjo en acciones constituidas como barreras para la ocurrencia de errores asistenciales, legitimando así su rol como corresponsables de la atención segura.

Descriptores: Seguridad del Paciente; Participación del Paciente; Atención Dirigida al Paciente; Prestación de Atención de Salud.

\section{INTRODUÇÃO}

O cuidado perioperatório tem acompanhado os avanços sociais, científicos e tecnológicos, constituindo desafio às organizações de saúde. Exige planejamento e adoção de práticas sistemáticas para o atendimento seguro do paciente durante o procedimento cirúrgico ${ }^{1}$.

A segurança do paciente, amplamente discutida atualmente, corresponde à redução, ao mínimo aceitável, do risco de dano desnecessário associado ao cuidado em saúde, sendo o evento adverso aquele incidente que resulta em dano ao paciente 2 .

As consequências das falhas nos serviços de saúde trazem impactos negativos tanto aos pacientes e suas famílias, quanto às organizações e sociedade. A ocorrência de eventos adversos no atendimento aos pacientes hospitalizados acarreta complicações na evolução de sua recuperação, aumento de taxas de infecções e do tempo médio de internação ${ }^{3}$. 
Diante disso, importante mudança nos paradigmas assistenciais tem sido discutida dando relevância à experiência da doença vivida pelo paciente e ao seu envolvimento como uma prioridade para a segurança no cuidado ${ }^{4,5}$. Nessa perspectiva, o cuidado centrado no paciente, atual modelo de assistência recomendado pela Organização Mundial da Saúde (OMS), promoveu um maior reconhecimento do envolvimento do paciente como um recurso crucial para a saúde, devido aos benefícios nos resultados clínicos e na sustentabilidade dos sistemas de saúde ${ }^{5,6}$.

O envolvimento do paciente relaciona-se ao processo de tomada de decisão sobre as questões de sua saúde, incluindo a participação ativa no planejamento, monitoramento e avaliação do cuidado ${ }^{7}$. Abrange níveis de participação/envolvimento de acordo com desejos e capacidades individuais, em parceria com profissionais e instituições, considerando os atributos de personalização, acesso, comprometimento e aliança terapêutica ${ }^{8}$. Na internação hospitalar aumenta a segurança da assistência e tem sido incentivado como forma de minimizar a ocorrência de incidentes ${ }^{9}$. Para que aconteça efetivamente é preciso que pacientes, cuidadores e familiares tenham conhecimento sobre segurança e ações que tornem o cuidado mais seguro.

Pacientes que relatam maiores níveis de participação no cuidado têm metade da taxa de incidentes relacionados à assistência, quando comparados àqueles com baixa participação ${ }^{10}$. Pacientes envolvidos apresentam maior adesão aos planos terapêuticos, maior satisfação, maior entendimento sobre sua condição de saúde e confiança na equipe assistencial e menos processos judiciais contra a instituição ${ }^{11}$.

Há escassez de estudos sobre as percepções do paciente sobre segurança e seu envolvimento no cuidado,12 em saúde durante o período de internação hospitalar, bem como sobre ações institucionais de incentivo à essa prática ${ }^{13,14}$.

Desvelar como esse movimento acontece nas instituições de saúde, pelo olhar do paciente, pode auxiliar no desenvolvimento de estratégias organizacionais que tornem o envolvimento do paciente no cuidado uma prática cultural.

O presente estudo teve como objetivo investigar a percepção do paciente cirúrgico sobre segurança do paciente e o seu envolvimento no cuidado à saúde durante a internação hospitalar.

\section{MÉTODO}

Realizou-se um estudo exploratório, de abordagem qualitativa, em um hospital de reabilitação do Distrito Federal, em unidade de internação cirúrgica ortopédica. A escolha dessa instituição se deu pelo perfil de atendimento ao paciente e princípios institucionais que consideram a pessoa como agente da sua própria saúde, aspecto fundamental ao envolvimento do paciente no cuidado à saúde.

A população foi constituída de pacientes adultos, sendo incluídos aqueles submetidos à cirurgia de artroplastia de quadril ou joelho. A seleção foi decorrente da complexidade dos procedimentos cirúrgicos que exigia permanência no ambiente hospitalar de sete dias, em média. Considerou-se que pacientes com maior tempo de internação teriam melhores condições de responder às questões do estudo, visto que, nesse período, os pacientes já tinham vivenciado experiências assistenciais de diferentes graus de complexidade no período pós-operatório e já estavam iniciando seu programa de reabilitação. Os critérios de exclusão foram o diagnóstico prévio de demência e a ocorrência de delirium no pós-operatório.

Durante o período do estudo, 192 pacientes foram internados na unidade de ortopedia, dos quais 18 foram submetidos à cirurgia de artroplastia de quadril e quatro à artroplastia de joelho. Três pacientes foram excluídos após aplicação dos critérios de exclusão, totalizando 19 pacientes elegíveis para o estudo.

A coleta de dados foi realizada por três enfermeiras, vinculadas a um grupo institucional de estudos em segurança do paciente, em setembro de 2016, utilizando a técnica de entrevista semi-estruturada. Utilizou-se roteiro com as seguintes perguntas: Quando eu falo o termo "Segurança do Paciente", quais pensamentos ou compreensões Ihe ocorrem sobre isso? O que você entende sobre "erros na assistência em saúde? Você acredita que esses erros ocorrem por quais motivos? Você se sente seguro no ambiente hospitalar? Por quê? Qual /quais atitudes você poderia ter para evitar um erro durante a internação hospitalar? O que os profissionais de saúde devem fazer para evitar ou minimizar os erros na assistência? O instrumento incluiu a caracterização do participante, com questões sobre idade, sexo, estado civil, nível de instrução, ocupação, internação e cirurgias prévias.

As entrevistas foram realizadas em ambiente privativo, gravadas, mediante autorização dos participantes. Pacientes que não aceitaram ter sua voz gravada, tiveram sua fala registrada por escrito. Cada entrevista durou cerca de 25 minutos.

A coleta de dados encerrou-se mediante saturação, ou seja, quando os dados obtidos nas entrevistas começaram a se repetir, deixando de trazer novos temas ${ }^{15}$. A amostra do estudo foi constituída por 14 pacientes, selecionados aleatoriamente. Dessa forma, cinco pacientes elegíveis não compuseram a população, devido ao critério de saturação. 
Para garantir sigilo quanto à exposição da identidade do participante, os relatos foram organizados numericamente por ordem de participação, atribuindo a letra E de entrevista, seguida de um número ordinal.

As entrevistas foram transcritas e analisadas à luz do referencial de análise de conteúdo, proposto por Bardin. Seguiram-se três etapas cronológicas: pré-análise, que consiste na escolha de documentos a serem analisados, a formulação das hipóteses e a elaboração de indicadores que fundamentem a interpretação final; exploração do material, quando se realiza a operação de codificação, cujo objetivo é alcançar o núcleo de compreensão do texto; e tratamento dos dados obtidos, onde o pesquisador irá alcançar inferências, realizando interpretações e confrontações com o material ${ }^{16}$

As unidades de significado foram definidas por temas. Os discursos revelaram oito unidades de significados que, pelo critério semântico, foram classificadas em duas categorias temáticas. A categoria 'Percepção do paciente sobre segurança do cuidado' constituiu-se a partir das unidades de significação 'Segurança do paciente relacionada às características organizacionais', 'Relação paciente-profissional e humanização da assistência como premissas para a segurança do paciente', 'Sentimento de segurança durante hospitalização', 'Reconhecimento dos erros em saúde'

A categoria 'Envolvimento do paciente no cuidado à saúde' revelou-se das unidades de significação 'Adesão do paciente aos protocolos institucionais', 'Colaboração do paciente nas estratégias institucionais de segurança do paciente', 'Participação do paciente na segurança do cuidado a partir da percepção dos riscos assistenciais' e 'Papel do profissional de saúde para engajar o paciente na segurança da assistência'.

A resolução do Conselho Nacional de Saúde no 466/2012 foi respeitada. Pesquisa aprovada por Comitê de Ética em Pesquisa, CAAE: 57224816.5.0000.0022. Todos os pacientes convidados aceitaram participar e assinaram o Termo de Consentimento Livre e Esclarecido.

\section{RESULTADOS}

Foram entrevistados 14 pacientes em pós-operatório de artroplastia de quadril ou de joelho, entre o quarto e sétimo dia de pós-operatório. As características dos participantes estão apresentadas na Tabela 1.

TABELA 1: Características dos participantes. Brasília, Brasil, 2016.

\begin{tabular}{lll}
\hline Variável & $\mathbf{n}$ & $\%$ \\
\hline Sexo & & \\
$\quad$ Feminino & 7 & 50 \\
$\quad$ Masculino & 7 & 50 \\
Estado civil & & \\
$\quad$ Casado & 8 & 57,1 \\
$\quad$ Solteiro & 3 & 21,4 \\
$\quad$ Divorciado & 3 & 21,4 \\
Escolaridade & & \\
Fundamental incompleto & 4 & 28,6 \\
Fundamental completo & 1 & 7,1 \\
Médio incompleto & 1 & 7,1 \\
Médio completo & 6 & 42,9 \\
Superior completo & 1 & 7,1 \\
Pós-graduação & 1 & 7,1 \\
Ocupação & & \\
Aposentado & 6 & 42,9 \\
$\quad$ Desempregado & 2 & 14,3 \\
Empregado & 4 & 28,6 \\
Outros & 2 & 14,3 \\
Internação prévia & & \\
Sim & 13 & 92,9 \\
$\quad$ Não & 1 & 7,1 \\
Cirurgia prévia & & \\
Sim & 11 & 78,6 \\
$\quad$ Não & 3 & 21,4 \\
Total & 14 & 100 \\
\hline
\end{tabular}

A idade dos pacientes variou de 31 a 79 anos, com média de 54 anos (DV= 13 anos). A maior parte dos entrevistados era casada. Quanto à escolaridade, predominou o ensino médio completo e quanto à ocupação, 
predominaram os aposentados. Apenas um paciente estava em sua primeira internação e três pacientes não possuíam histórico de cirurgias anteriores.

Após a análise de conteúdo das entrevistas, emergiram duas categorias temáticas: Percepção do paciente sobre segurança do cuidado e Envolvimento do paciente no cuidado à saúde.

\section{Percepção do paciente sobre segurança do cuidado}

Ao serem questionados sobre o termo segurança do paciente, os participantes revelaram que a segurança do cuidado está presente quando os cuidados em saúde não causam danos. Associaram segurança do paciente às características organizacionais, incluindo a limpeza do ambiente, estrutura física adequada, equipe assistencial capacitada, assim como possuir confiança na instituição também foram aspectos revelados pelas narrativas.

Segurança do paciente, eu acho que é não cair de uma cama, ter o atendimento na hora certa. (E5)

Acidentes pós-cirúrgicos tipo infecção hospitalar. É isso que me vem na cabeça. (E6)

Segurança do paciente é [...] você se sentir segura dentro do hospital. A cama segura e todo mobiliário que você vai usar, que sejam seguros. Tudo isso pra mim é segurança. Segurança nas salas de cirurgia, médicos competentes. (E3)

Melhor hospital da América Latina. Esperei dois a três anos para fazer uma cirurgia aqui. Sinto muita segurança aqui. (E4)

A relação entre paciente-profissional, com destaque à humanização do cuidado, ficou evidente em alguns relatos como aspectos relacionados à segurança na assistência.

No meu modo de pensar, é começando pela atenção do cuidador que tá cuidando do paciente. Pela atenção, pelo carinho, pela responsabilidade [...] e amor também. (E11)

É um tipo de zelar pelo paciente. Porque a gente fica aqui, às vezes, sem defesa, né? (E13)

Quanto à percepção do paciente em relação à segurança durante a sua hospitalização, os discursos revelaram um sentimento positivo em decorrência da confiança que sentem nos profissionais de saúde e da organização do serviço.

Eu acho que aqui a gente está cercado de pessoas que estão aqui para te ajudar né, não para fazer o mal pra você né? E se acontecer algum erro é porque houve um erro mesmo, não é intenção da pessoa fazer isso. Então por isso eu me sinto seguro. (E1)

Ah, porque aqui tem organização. É bem organizado. O pessoal atende a gente aqui toda hora. É tudo limpinho, tudo esterilizado. (E5)

Assim como em relação à segurança do paciente, foi observada certa dificuldade em elaborar um conceito para os erros em saúde. Entretanto os participantes reconhecem que esses erros podem acontecer. Os eventos adversos citados por eles foram quedas, infecção, erros de medicação e de lateralidade cirúrgica. Os discursos revelaram que a percepção foi construída a partir de situações vivenciadas por eles ou veiculadas pelos meios de comunicação.

Depois da cesárea, eu comecei a sentir muitas dores. [...] Faz outro exame: "Ah é gravidez tubária ou um cisto na trompa. Interna lá e opera". Ai ele (o médico) falou que tinha tirado um pedaço da trompa, mas que eu poderia ter outro neném. Mentira das boas porque eu comecei a passar mal outra vez e ele me mandou pro hospital do câncer para fazer radioterapia. Ai quando fez o primeiro exame lá, falaram assim "ele deixou o negócio apodrecer [...] e você nunca mais vai ter outro filho" Foi um erro gravíssimo. Eu tenho meu filho lindo e maravilhoso, mas eu poderia ter tido mais né? (E3)

Eu vejo umas reportagens que passam na televisão. É pra operar o direito e opera o esquerdo. Já vi isso acontecer muito em reportagem (E12)

Para os pacientes, a ocorrência de erros em saúde está associada à dinâmica do processo de trabalho, treinamento e comportamento do profissional.

Basicamente tudo quanto é erro é o treinamento da pessoa. E o treinamento envolve tudo né: a atenção do funcionário, do especialista na área, tem que ter esse treinamento com ele. (E1)

Eu acho que por muitas vezes por desatenção né da pessoa, quando a pessoa não tem atenção, não tem cuidado. (E2)

Excesso de trabalho, muita movimentação grande. Estresse né? Às vezes a pessoa tá estressada, porque aqui nesse hospital vocês trabalham muito. (E5)

Para alguns pacientes a percepção de segurança e dos erros em saúde não estava muito clara, mas demonstraram interesse em saber mais sobre o tema.

Então erro, não saberia te dizer agora não. (...) Eu não me atentava para isso. Segurança de cada paciente que está internado é um assunto realmente interessante por isso eu acho que resolvi conversar, até mesmo para saber sobre o assunto. (E10) 


\section{Envolvimento do paciente no cuidado à saúde}

Ao questionar sobre o que podem fazer para evitar que os erros aconteçam, a percepção de parte dos pacientes converge para a adesão dos protocolos institucionais.

Seguir as regras que o hospital tá te impondo. Aqui, tomar o banho certinho antes da cirurgia, fazer os exames certos, a medicação certa, ter todo o cuidado, seguir ao pé da letra o que o profissional da saúde tá te pedindo pro trabalho dele ser bem-sucedido. (E8)

As narrativas evidenciaram que o paciente reconhece estratégias instituicionais para aumentar a segurança do paciente. $O$ processo de identificação do paciente durante a administração de medicamentos foi reconhecido como forma de evitar erros.

Eu vi mesmo essa semana que tinham duas pessoas com o mesmo nome e sobrenome. A preocupação de se atentar mais ainda em relação a isso pra não acontecer o erro né?! Porque coincidindo nomes iguais ou semelhantes, é um possível problema de erros mesmo onde vocês têm uma responsabilidade ainda maior porque a medicação vai ser diferente. (E10)

Na percepção dos pacientes, o processo de administração de medicamentos é uma das atividades com maior potencial para a ocorrência de erros. Os pacientes se mostraram apreensivos com medicamentos desconhecidos, com a possibilidade de tomar medicamentos de outro paciente, de horários diferentes dos habituais, possíveis reações após a administração de medicamentos e medicamentos que não surtem o efeito desejado.

Uma coisa, por menor que seja, possa prejudicar, por exemplo, um remédio pra dor de cabeça que faça mal ao meu estômago. (E7)

Antes de acontecer o erro a gente pode orientar alguma coisa. Eu não tava dando certo com o comprimido, então eu avisei pra ela antes. (E5)

Em decorrência desse potencial, se envolvem na assistência a fim de prevenir possíveis incidentes, assumindo um papel ativo no processo de tornar o cuidado mais seguro.

Tem que perguntar também né o que tá tomando, igual eu pergunto. Eu pergunto que remédio é esse? Pra que que é? (E2)

$O$ remédio que eu tava tomando pra pressão era de 12 em 12 horas. Eu notei uma falhazinha e eu reclamei porque não tava dando. Às vezes ultrapassava aquele horário e eu sou enjoada nisso, entende? (E3)

Nessa mesma perspectiva, as narrativas evidenciaram que a preocupação que eles têm em relação aos procedimentos a que são submetidos aumenta, especialmente, ao perceberem a existência de um potencial incidente. Assim, um participante trouxe um relato de uma experiência vivenciada em outra instituição de saúde, em que se envolveu no cuidado, ao alertar a equipe sobre o erro e, assim, contribuiu com a segurança da assistência.

Na hora que eu ia entrando no centro cirúrgico, me perguntaram é o olho direito não é? E eu disse: é o esquerdo. Se não pergunta, poderiam operar o olho bom. Elas falaram: é o olho direito que tá marcado aqui. Eu disse que era o esquerdo e comecei a ficar nervoso. (E12)

Para os pacientes, a forma com que a informações sobre diagnóstico e tratamento são repassadas podem despertar o interesse e auxiliar no melhor entendimento dele sobre o seu papel na segurança do cuidado a ser prestado. Nesse processo, o profissional de saúde é um elemento-chave no incentivo ao envolvimento do paciente.

Alguns profissionais deveriam ajudar o paciente a conhecer a própria doença. Não achar que o profissional sabe tudo. (E5)

\section{DISCUSSÃo}

O conhecimento sobre segurança do paciente foi impreciso do ponto de vista conceitual. Entretanto, os entrevistados fizeram relações pertinentes ao tema, relacionando a segurança do paciente à assistência livre de danos, qualidade da assistência e humanização do cuidado.

Os pacientes sentiam-se seguros no hospital durante aquela internação e justificaram citando aspectos como limpeza do ambiente, mobiliário adequado e equipe capacitada. Opiniões semelhantes foram encontradas em estudo realizado no Brasil que investigou os atributos de satisfação relacionados à segurança e qualidade. Nesse estudo, os temas relacionados à segurança e qualidade que emergiram da experiência dos pacientes e familiares foram estruturais, como o ambiente adequado e recursos disponíveis para o tratamento e associados ao processo de cuidado como a competência técnica dos profissionais e a interação entre os pacientes e a equipe assistencial ${ }^{17}$.

Os participantes que não tinham aproximação com o tema manifestaram interesse em saber mais. Compartilhar informações, envolver os pacientes na avaliação e planejamento de cuidados são atividades que promovem a participação do paciente ${ }^{18}$. Sugere-se que os profissionais de saúde estabeleçam boa comunicação com os pacientes e busquem incluí-los no processo de cuidado. 
Apresentaram dificuldade em elaborar uma resposta ao serem questionados quanto aos erros na assistência em saúde. Muitos citaram casos graves de erros na assistência à saúde noticiados pelos meios de comunicação. Isso mostra que precisam ser orientados para observarem os possíveis incidentes durante seu próprio cuidado e não apenas aqueles que levam a incapacidades e óbito.

O principal tipo de erro assistencial relatado foram os erros de medicação. Alguns entrevistados citaram a medicação como uma atividade potencial para ocorrência de erros e, por isso, tendem a adotar uma postura mais ativa perante esse cuidado. Atitudes como questionar os profissionais sobre os medicamentos e estar atento quanto ao procedimento de identificação do paciente na administração de medicamentos foram exemplos mencionados pelos participantes.

Ao fornecer informações ao paciente sobre a indicação do medicamento, o efeito esperado e possíveis efeitos adversos, os profissionais de saúde oferecem subsídios para que ele atue como barreira contra os erros relacionados às medicações. A equipe de enfermagem como responsável pela administração de medicamentos, deve aproveitar esse momento para orientar o paciente e incentivar seu envolvimento ${ }^{19}$

A maior parte dos pacientes manifestaram interesse pela segurança do paciente e reconheceram a importância de seu envolvimento nesse processo. Isso converge com o desejo dos pacientes em ter um papel ativo no seu cuidado e serem considerados parceiros da equipe assistencial ${ }^{4}$.

A participação do paciente no seu próprio cuidado demanda mudança cultural nos serviços de saúde para que os profissionais possam estimular a autonomia dos pacientes e apoiar atitude ativa, considerando-os como parceiros e corresponsáveis por sua saúde e cuidados ${ }^{20}$.

A aproximação do profissional de saúde junto ao paciente é fundamental para inseri-lo em seu próprio cuidado. Nesse processo destaca-se a comunicação que deve ser cada vez mais aberta e horizontal, de modo a minimizar possíveis relações de poder e fortalecer a tomada de decisão compartilhada ${ }^{20,21}$.

No cuidado centrado na pessoa, o paciente é visto como especialista sobre si mesmo e considerado como parceiro da equipe de saúde 4 . Ouvir e valorizar a opinião dos pacientes, compreendendo suas vivências e expectativas e considerando suas preferências é o caminho a seguir para reconhecer o envolvimento do paciente como elemento chave da melhoria da qualidade do cuidado 22 .

Busca-se, assim, alinhar o conhecimento científico à atuação do profissional de saúde, em consonância com as demandas atuais da sociedade. Os serviços de saúde devem valorizar e estimular o desenvolvimento de sua equipe, assim como fortalecer a gestão organizacional, a fim de promover o envolvimento do paciente na segurança da assistência, com reflexo na cultura institucional.

\section{Limitações do estudo}

As limitações do estudo estão relacionadas ao método de obtenção dos dados, uma vez que entrevistas podem inibir os participantes de expressarem suas reais percepções. Considera-se, porém, que não houve prejuízo ao alcance do objetivo proposto.

\section{CONCLUSÃO}

Os pacientes demonstraram limitações conceituais sobre a segurança do paciente, mas apresentaram relações pertinentes ao tema quando o associaram ao controle do risco de dano, à qualidade do cuidado e à humanização da assistência. A percepção de erro foi associada às ocorrências vivenciadas e observadas na mídia.

A participação dos pacientes no cuidado se deu de forma incipiente, uma vez que esteve, frequentemente, atrelada às preocupações dos procedimentos prescritos. Eles revelaram ações que configuraram barreiras para a ocorrência de erros relacionados à assistência à saúde, legitimando o seu papel como corresponsável pelo cuidado.

Conclui-se que o envolvimento do paciente no cuidado não é uma ação habitual, uma vez que não existe um padrão de comportamento do paciente em relação ao tema. Incorporar à cultura organizacional e capacitar os profissionais de saúde para o desenvolvimento de ações e protocolos de admissão que envolvem o paciente no cuidado é necessário.

Considera-se que o estudo possibilitou identificar a necessidade de estabelecer estratégias para envolver o paciente cirúrgico no cuidado, a fim de aumentar sua própria segurança durante o processo de hospitalização. Além disso, mostrou que é preciso trabalhar as competências dos profissionais de saúde para incluírem, em sua prática cotidiana, o incentivo ao envolvimento do paciente no seu próprio cuidado.

O desenvolvimento de competências pode minimizar possíveis divergências entre o comportamento ativo dos pacientes e do profissional de saúde. Fortalecer a cultura de segurança do paciente incluindo, nesse processo, a alta gestão das instituições de saúde, pode favorecer a mudança comportamental de pacientes, familiares e profissionais de saúde para um efetivo envolvimento do paciente no cuidado. 


\section{REFERÊNCIAS}

1. Batista J, Cruz EDA, Alpendre FT, Rocha DJM, Brandão MB, Maziero ECS. Prevalence and avoidability of surgical adverse events in a teaching hospital in Brazil. Rev. Latino-Am. Enfermagem [Internet], 2019 [cited 2020 Jun 10]; 27:e2939. DOI: https://doi.org/10.1590/1518-8345.2939.3171.

2. World Health Organization. World Alliance for Patient Safety: the conceptual framework for the international classification for patient safety. Geneva, 2009. [cited 2020 May 01] Available from: http://www.who.int/patientsafety/taxonomy/icps_full_report.pdf.

3. Agência Nacional de Vigilância Sanitária. Assistência Segura: Uma Reflexão Teórica Aplicada à Prática Agência Nacional de Vigilância Sanitária. Brasília: Anvisa, 2017. [cited 2020 May 01] Available from: https://www20.anvisa.gov.br/segurancadopaciente/index.php/publicacoes/item/caderno-1-assistencia-segura-uma-reflexaoteorica-aplicada-a-pratica.

4. Ringdal M, Chaboyer W, Ulin K, Bucknall T, Oxelmark L. Patient preferences for participation in patient care and safety activities in hospitals. BMC Nurs. [Internet], 2017 [cited 2018 Apr 20]; 16:69. DOI: https://doi.org/10.1186/s12912-017-0266-7.

5. Graffigna G, Barello S. Patient Health Engagement (PHE) model in enhanced recovery after surgery (ERAS): monitoring patients' engagement and psychological resilience in minimally invasive thoracic surgery. Journal of Thoracic Disease [Internet], 2018 [cited 2019 Mar 21]; 10(Suppl 4):S517-28. DOI: https://doi.org/10.21037/jtd.2017.12.84.

6. Barello S, Graffigna G, Vegni E, Savarese M, Lombardi F, Bosio AC. Engage me in taking care of my heart: a grounded theory study on patient-cardiologist relationship in the hospital management of heart failure. BMJ Open. [Internet], 2015 [cited 2018 Oct 18]; 5:e005582. Available from: https://bmjopen.bmj.com/content/5/3/e005582.

7. Sahlström M, Partanen $P$, Rathert C, Turunen H. Patient participation in patient safety still missing: Patient safety experts views. Int. J. Nurs. Pract. [Internet], 2016 [cited 2018 Oct 18]; 22(5):461-9. Available from: https://pubmed.ncbi.nlm.nih.gov/27507224/.

8. Higgins T, Larson E, Schnall R. Unraveling the meaning of patient engagement: a concept analysis. Patient Educ. Couns. [Internet], 2017 [cited 2020 Jun 13]; 100:30-6. DOI: https://doi.org/10.1016/j.pec.2016.09.002.

9. Rather C, Brandt J, Williams ES. Putting the 'patient' in patient safety: a qualitative study of consumer experiences. Health expect. [Internet], 2012 [cited 2018 Oct 18]; 15(3):327-36. Available from: https://pubmed.ncbi.nlm.nih.gov/21624026/.

10. Weingart SN. et al. Hospitalized patients' participation and its impact on quality of care and patient safety. Int. J. Qual. Health Care [Internet], 2011 [cited 2018 Oct 5]; 23(3):269-77. DOI: https://doi.org/10.1093/intqhc/mzr002.

11. Schank EC, Bryant RA, Van Son CR, Odom-Maryon T. Perspectives on patient and family engagement with reduction in harm: the forgotten voice. J. Nurs. Care Qual. [Internet], 2018 [cited 2018 Oct 5]; 34(1):73-9. DOI: https://doi.org/10.1097/NCQ.0000000000000333.

12. Spruce L. Back to Basics: Patient and Family Engagement. AORN J. [Internet], 2015 [cited 2017 Aug 12]; 102(1): 33-9. DOI: http://dx.doi.org/10.1016/j.aorn.2015.04.020.

13. Schawappach DLB, Frank O, Buschmann U, Babst R. Effects of an educational patient safety campaign on patients' safety behaviors and adverse events. J. Eval. Clin. Pract. [Internet], 2013 [cited 2018 Oct 28]; 19(2):285-91. DOI: https://doi.org/10.1111/j.1365-2753.2012.01820.x.

14. Figueiredo FM, Gálvez AMP, Garcia EG, Eiras M. Participation of patients in healthcare security: a systematic review. Ciênc. saúde coletiva [Internet], 2019 [cited 2020 Jun 12]; 24(12):4605-20. DOI: https://doi.org/10.1590/1413812320182412.08152018

15. Cavalcante RB, Calixto $P$, Pinheiro MMK. Análise de Conteúdo: considerações gerais, relações com a pergunta de pesquisa, possibilidades e limitações do método. Inf soc - estud. [Internet], 2014 [cited 2017 Nov 15]; 24(1):13-8. Available from: https://periodicos.ufpb.br/ojs2/index.php/ies/article/view/10000.

16. Bardin L. Análise de conteúdo. Tradução de Luís Antero Reto e Augusto Pinheiro. São Paulo: Edições 70, 2016.

17. Costa DG da, Moura GMSS de, Moraes MG, Santos JLG dos, Magalhães AMM de. Satisfaction attributes related to safety and quality perceived in the experience of hospitalized patients. Rev. Gaúcha Enferm. [Internet]. 2020 [cited 2020 Apr 28]; 41(spe):e20190152. Available from: http://www.scielo.br/scielo.php?script=sci_arttext\&pid=S1983$14472020000200404 \&$ Ing=en

18. Tobiano G. et al. Patient participation in nursing care on medical wards: an integrative review. Int. J. Nurs. Stud. [Internet], 2015 [cited 2019 Apr 10]; 52(6):1107-20. DOI: https://doi.org/10.1016/j.ijnurstu.2015.02.010.

19. Mctier L, Botti M, Duke M. Patient participation in medication safety during an acute care admission. Health Expect. [Internet] 2015 [cited 2018 Oct 20]; Oct; 18(5): 1744-56. DOI: https://doi.org/10.1111/hex.12167.

20. Silva T de O, Bezerra ALQ, Paranaguá TT de B, Teixeira CC. Patient involvement in the safety of care: an integrative review. Rev. Eletr. Enferm. [Internet]. 2016 [cited 2017 Dec 09]; 18:e1173. Available from: https://revistas.ufg.br/fen/article/view/33340.

21. McCormack L, Thomas V, Lewis MA, Rudd R. Improving low health literacy and patient engagement: A social ecological approach. Patient Educ Couns [Internet], 2017 [cited 2020 Jun 09]; 100(1):8-13. DOI: https://doi.org/10.1016/j.pec.2016.07.007.

22. Graffigna, G. et al. Measuring patient engagement: Development and psychometric properties of the Patient Health Engagement (PHE) scale. Frontiers in Psychology [Internet], 2015 [cited 2020 Jun 09]; 6:274. DOI: https://doi.org/10.3389/fpsyg.2015.00274. 